\title{
The Liquisolid technique: an overview
}

\author{
Shashidher Burra*, Madhusudhanrao Yamsani, Venkateswarlu Vobalaboina
}

\author{
Department of Pharmaceutics, University College of Pharmaceutical Sciences, \\ Kakatiya University, Warangal, Andhra Pradesh, India
}

\begin{abstract}
A novel "Powder Solution Technology" involves absorption and adsorption efficiency which makes use of liquid medications, drug suspensions admixed with suitable carriers, coating materials and formulated into free flowing, dry looking, non adherent and compressible powder forms. Based upon a new mathematical model expression, improved flow characteristics and hardness of the formulation has been achieved by changing the proportion of Avicel ${ }^{\circledR}$ PH 200 and Aerosil ${ }^{\circledR}$ PH 200 from 50:1 ratio to 5:1 and in which the drug is dispersed in an almost molecularly state. Due to their significantly improved wetting properties a greater drug surface area is exposed to the dissolution media, resulting in an increased dissolution rate and bio availability. By using the Liquisolid technique, sustained drug delivery systems were developed for the water soluble drugs in which hydrophobic non-volatile solvents are used as vehicles.
\end{abstract}

Uniterms: Liquisolid. Solubility. Bioavailability. Flow properties. Load factor. Powder solution technology.

A nova "Tecnologia da Solução Sólida" envolve eficiência de absorção e de adsorção, faz uso de medicações líquidas, suspensões de fármacos e misturas com transportadores adequados, materiais de cobertura e é formulada em formas sólidas em fluxo livre, secas, não aderentes e compressíveis. Com base em novo modelo matemático, características aprimoradas de fluxo e dureza da formulação foram alcançadas modificando-se a proporção de Avicel ${ }^{\circledR}$ PH 200 e Aerosil ${ }^{\circledR}$ PH 200 de 50:1 para 5:1, na qual o fármaco é disperso quase que no estado molecular. Devido às propriedades de umidificação significativamente aprimoradas e à área do fármaco exposta ao meio de dissolução, que resulta na velocidade de dissolução, a biodisponibilidade foi aumentada. Utilizando a técnica Liquisólido, desenvolveram-se sistemas de liberação controlada de fármacos solúveis em água, nos quais solventes hidrofóbicos não voláteis foram usados como veículos.

Unitermos: Liquisólido. Solubilidade. Biodisponibilidade. Propriedades de fluxo. Fator de carga. Tecnologia de solução sólida.

\section{INTRODUCTION}

The poor dissolution characteristics of water insoluble drugs are a major challenge for pharmaceutical science. Powder solutions are designed to contain liquid medications in powdered form and there have been numerous advances in increasing drug dissolution rate profiles. During the past few years many techniques have been developed such as drug micronization, solid dispersions, coprecipitation, lyophilization, micro- encapsulation, use of pro-drug and drug derivatization processes, and inclusion of drug solutions into soft gelatin capsules (Kapsi, 2001).

*Correspondence: Shashidher Burra. University College of Pharmaceutical Sciences, Kakatiya University, Department of Pharmaceutics, Warangal, Andhra Pradesh, India. E-mail: shashidher85@gmail.com
Micronization is the most common method used to increase the surface area of the drug, but this becomes less effective when they are formulated as tablets or encapsulations (Aguiar et al., 1979; Finholt, 1968; Lin et al., 1968). In the case of soft gelatin capsules, Ebert's (1977) review stated that these products demonstrated the most efficient bio availability since the drug is already in solution form. However, the formulation of soft gelatin capsules is expensive and requires sophisticated technology. Nevertheless, best approaches are available to prepare the liquid oily medications and drug solutions of water insoluble solid drugs.

Bio-Pharmaceutical classification class II (water insoluble drugs), the rate of oral absorption is often controlled by the rate in the GIT. Therefore, together with 
permeation, solubility profile and dissolution rate profiles of drugs are major key factors for its oral bio-availability. The concept of powdered solutions makes it possible to convert drug solutions or liquid medications into moderately flowing powders by an admixture with selected powder excipient. Some investigators have used a similar approach to increase release profiles of several water-insoluble drugs (Spireas, 2002).

A latest contemporary technique, authorized "Powder Solution Technology" has been implemented to prepare water-insoluble drugs into prompt release solid dosage forms. The Liquisolid techniques are considered as pleasantly flowing and compressible powdered forms of liquid medications. These liquid medications may be regenerated into dry - looking or moistureless, nonadherent free-flowing and readily compressible powders by a simple admixture with selected carriers and coating materials. In this technique, the drug might be in solid dosage form and dissolved in solution or a solvent. Drug is solubilized in a maximum molecularly dispersed state. Therefore, this is due to their significantly increased wetting properties and increased surface area of drug available for dissolution. Water- insoluble or poorly water soluble drugs may be expected to have increased dissolution rate properties as well as improved bioavailability.

The Liquisolid compacts have two major formulation components namely, powder substrate and liquid medication. The powder substrate mainly consists of: a) compression enhancing, large preferably porous carrier particles; b) flow enhancing, very fine, highly adsorptive coating material particles. Liquisolid preparations may be hampered by their poor and erratic flow and compaction properties. Inherently, the acceptable flow and compaction characteristics of the finished powder / liquid admixtures are secured.

The quantities of various excipients required for powder solution formulations are determined in accordance with a new mathematical model expression (Liao, 1983). The absorbate molecules diffuse inside the absorbent and are eventually captured by the powder particles within their bulk, and thus absorption of the liquid occurs. Adsorption is the phenomenon in which liquid is not truly absorbed and instead of being dispersed through out the interior of the solid, the molecules only cling to its available surface, internal and external. However, depending on the sorbent properties, both of these processes can occur simultaneously, and is termed sorption. Initially the liquid is absorbed in the interior of the particles captured by its internal structure and after saturation of this process adsorption of the liquid onto the internal and external surfaces of the porous carrier particles occurs (Rania et al., 2008).

\section{DEFINITIONS}

Liquid medication: liquid lipophilic drugs and drug suspensions or solutions of solid water - insoluble drugs in suitable non volatile solvent systems are called Liquid medication.

Solubility: water insoluble drugs include those drugs that are "sparingly water soluble" (one part solute into 30-100 parts of water), slightly water- insoluble (one part solute into $1000-10,000$ parts of water) and practically "water-insoluble" or insoluble (one part solute into 10,000 or more parts of water).

The liquisolid technique systems refers to powdered forms of liquid medications formulated by changing to liquid lipophilic drugs or drug suspensions or solutions of water insoluble solid drugs in suitable non-volatile solvent systems into dry-looking, non-adherent, free moderately flowing.

Liquisolid systems based on the type of liquid medication can be classified into three sub groups:

(i) "Powdered drug solutions"

(ii) "Powdered drug suspensions"

(iii)"Powdered liquid drug"

The first two groups may exist or be produced by changing drug solutions and drug suspensions while the third is produced from the formulation of liquid drugs into liquisolid systems.

“Liquisolid compacts": refers to immediate sustained-release tablets or capsules that are described under "liquisolid systems".

"Liquisolid Microsystems": refers to capsules prepared by "liquisolid systems" plus the inclusion of an additive resulting in a unit size that may be as much as five times less than that of a liquisolid compact.

Liquid load factor $\left(\mathrm{L}_{\mathrm{f}}\right)$ : defined as the ratio of the amount of liquid medication (W) over the quantity of carrier material (Q) in the system.

$\mathrm{L}_{\mathrm{f}}=\frac{\mathrm{W}}{\mathrm{Q}}$

Carrier: Coating Material Ratio (R): Ratio between the quantities of carrier $(\mathrm{Q})$ and coating materials (q) present in the formulation.

$\mathrm{R}=\frac{\mathrm{Q}}{\mathrm{q}}$

"Carrier material": refers to a preferably porous material possessing sufficient absorption properties.

“Coating Material”: refers to a material possessing fine and highly adsorptive particles. 


\section{ADVANTAGES}

1) Large number of Bio - Pharmaceutical classification class 2 drugs with high permeability, slightly or very slightly water soluble and practically insoluble liquids and solid drugs can be formulated into Liquisolid systems.

2) Improvement of Bio-availability of an orally administered water insoluble drugs is achieved

3) This principle governs or administers the mechanism of drug delivery from liquisolid systems of powdered drug solutions and it is mainly responsible for the improved dissolution profiles exhibited by this preparations.

4) Drug is formulated in a tablet form or encapsulated dosage form and is held in solubilized liquid state, which confers developed or improved drug wetting properties thereby improving drug dissolution profiles.

5) In this technique, production cost is low compared to soft gelatin capsules.

6) This liquisolid system is specifically for powdered liquid medications.

7) Greater drug surface area is exposed to the dissolution medium.

8) These Liquisolid systems formulate into immediate release or sustained release dosage forms.

9) Optimized sustained release Liquisolid tablets or capsules of water insoluble drugs demonstrate constant dissolution rates (Zero Order Release).

\section{DISADVANTAGES}

1) This technique is only for water insoluble drugs.

2) However, for formulation of high dose insoluble drugs the liquisolid tablet is one of the limitations of this technique.

3) In order to achieve acceptable flow ability and compactability for liquisolid powder formulation, high levels of carrier material and coating materials should be added. This will increase the weight of tablets to above one gram which makes them difficult to swallow. Therefore, it is impossible with conventional tablet methods to convert high dose to liquisolid tablets with a tablet weight of less than50 mg. Dissolution profile enhancement occurs in the presence of low levels of hydrophilic carrier, where coating material is not significant.

\section{FORMULATION DESIGN}

Non-Volatile solvents: Poly Ethylene Glycol (PEG)-400. PEG- 200, PEG- 4000, PEG- 6000, Polysorbate80, Tween-80, Propylene Glycol (PG) etc.
Carriers: Avicel ${ }^{\circledR}$ RTM 105, Avicel ${ }^{\circledR}$ PH 102 granular Microcrystalline cellulose (MCC) grade, Avicel ${ }^{\circledR} \mathrm{PH}$ 200 coarse granular MCC grade, experimental grade of granular amorphous cellulose (E.G.C) starch, lactose used as carrier materials. Silica possessing large surface areas and MCC of fine particle size granular grades produced good flow properties and compression properties resulting in applicable tablets.

Super Disintegrates: Sodium starch glycolate (SSG), Crospovidone, Sodium croscarmellose .

Coating Materials: Aerosi $1^{\circledR} \mathrm{PH} 200$, Colloidal silica, Cab-O-sil RTM M5 used as coating material. By adding PVP to liquid medication, it would be possible to produce dry powder formulations containing liquid with a high concentration of drug.

The active pharmaceutical ingredient in a solid dosage form must undergo dissolution before it is available for absorption from the gastro intestinal tract. The dissolution rate is most often the rate-determining step in passive drug absorption. Liquisolid system demonstrated for drugs which would enhance the dissolution profile and the absorption capacity of water insoluble solid drugs such as digoxin, digitoxin, prednisolone, hydrocortisone, famotidine, carbamazepine, water insoluble vitamins, hydrochlorothiazide, Domperadone, spiranolactone, Carvedilol, Naproxen, and Meloxicam

\section{GENERAL PROCEDURE FOR LIQUISOLID TABLETS}

The Liquisolid tablet preparation method involves, first a mathematically calculated amount of pure drug weighed and dissolved in the suitable amount of solvent in a molecularly dispersed state. For attaining good flow properties trial and error methods were used i.e. changing the carrier : coating material ratio from 50:1 to 5:1 ratios according to new mathematical model expressions proposed by Liao. This liquid medication is poured on the suitable amount of carrier material. The liquid medication is absorbed into the carrier material internally and externally and then a suitable disintegrant was added to this material. Finally, coating material was added for dry looking, adherent to the carrier material for achieving good compression properties. Liquid medication is incorporated into carrier material which has a porous surface and closely matted fibers in its interior as cellulose. Both absorption and adsorption take place, i.e. first, the liquid absorbed into the interior of the particles is captured by its internal structure, and after saturation of this process, adsorption of the liquid onto the internal and external surface of the porous carrier particles occurs. A large surface area and 
high absorptive properties of the coating material gives the Liquisolid system the particular flow properties described (Martin et al., 1983; Spireas et al., 1992).

Solubility Studies: Solubility studies were conducted for the selection of high solubility of the pure drug form in the non volatile solvents, This involves pure drug dissolved in different non-volatile solvents. Excess amounts of pure drug were added to the non-volatile solvents, followed by saturation solution transfer to a rotatory shaker for 48 hours at $25^{\circ} \mathrm{c}$ under constant vibration. After a 48-hour period the saturated solution was filtered through a $0.45 \mu \mathrm{m}$ Millipore filter and analyzed.

\section{BULK PROPERTIES}

Rania et al. (2008) suggested pre compression studies of the Liquisolid powder systems. In the study, he suggested flowability of a powder is of major importance in the production of pharmaceutical dosage forms in order to attain a uniform feed and reproducible filling of tablet dies. The following parameters describe a measure of the flow properties of liquisolid systems that will be selected and compressed into tablets.

Angle of Repose Method: In this method, a fixed height cone method procedure was performed in triplicate and average angle of repose calculated (Rania et al.,2008).

Angle of repose $\operatorname{Tan} \theta=\frac{(\mathrm{h})}{\mathrm{r}}$

Bulk Density: This procedure involves the fixed height of each liquisolid powder substrate with prepared and fixed weight. This powder was placed in a graduated cylinder and the powder occupation volume measured and initial bulk density $\mathrm{D}_{\mathrm{B} \text { min }}$ calculated. The graduated cylinder was then allow to tap at constant velocity until a constant volume is obtained, when the powder was considered to have reached the most stable arrangement, the final bulk volume was recorded. Final bulk density $\mathrm{D}_{\mathrm{B} \max }$ was then calculated. (Rania et al.,2008).

Carr's index $\%=\frac{D_{B \text { max }}-D_{B \text { min }}}{D_{B \text { max }}} \times 100$

"Hausner" s ratio was calculated from the equation

"Hausner's" ratio $=\frac{D_{B \max }}{D_{B \text { min }}}$

Angle of Slide: For the determination of angle of slide, an approximately weighed amount of coating material was placed at one end of a metal plate with a polished surface. This end was raised gradually until the plate formed an angle with the horizontal at which the powder was about to slide. This angle $\theta$ represents the angle of slide. It was taken as a measure for the flow characteristics of powders. An angle of slide of $33^{\circ}$ corresponds to optimal flow properties (Spireas et al., 1999).

Contact Angle: For assessment of wettability, contact angle of Liquisolid tablets was measured according to the imaging method. To measure the contact angle, a drop of liquid is directly placed on a flat surface of the solid in the-so-called imaging method. The liquid drop is prepared using a saturation solution of the drug in Simulated Gastric Fluid, Simulated Intestinal Fluid media and an excessively large amount of pure drug was added to this media, shaken for 24 hours at a constant rate then the upper solution was centrifuged. A drop of this solution was placed on the surface of the tablet, and pictures were taken using a digital camera. By measuring the height and diameter of the sphere drop on the (Spireas et al., 1999) liquisolid tablets and direct compressed tablets, contact angle was measured. Liquisolid tablet contact angle is less than that of direct compressed tablets. Polysorbate 80 showed the lowest contact angle in the liquisolid tablets.

\section{Dissolution studies on Liquisolid tablets}

This entails the optimization of the hardness of the Liquisolid tablet, without applying excessive compression force yet assuring rapid disintegration and dissolution. In other words, tablets should be sufficiently hard to resist breaking during normal handling and yet quickly disintegrate properly after swallowing.

Dissolution rate $\left(D_{R}\right)$ is explained according to the "Noyes - Whitney" equation and "diffusion layer model" dissolution theories.

$D_{R}=(D / h) S(C s-C)$

According to this equation, stagnant diffusion layer thickness is $h$, and formed by the dissolving liquid around the drug particles. $\mathrm{D}$ is the diffusion coefficient of the drug molecules transported through it, $\mathrm{S}$ is the surface area of the drug available for dissolution, $\mathrm{C}$ is the drug concentration in the bulk of the dissolving medium, Cs is the saturation solution of the drug in the dissolution medium. Dissolution tests for liquisolid tablets were done at constant rotational speed and in identical dissolution media, thus allowing estimation of the thickness of the stagnant diffusion layer (h). From this equation, dissolution rate is directly proportional not only to the concentration gradient of the drug in the stagnant diffusion layer (Cs- C), but also to its surface area (S) available for dissolution. 
For estimation and comparison, drug dissolution rates $\left(D_{R}\right)$ of drug were used, with amount of drug dissolved per min presented by each tablet formulation during the first 10 minutes.

$\mathrm{D}_{\mathrm{R}}=\frac{(\mathrm{Mx} \mathrm{D})}{1000}$

Where, $\mathrm{M}$ is the total amount of pure drug in each tablet and D is the percentage of drug dissolved in the first 10 minutes (Spireas et al., 1998)

Importance of carrier and coating material ratio(R): Liquisolid systems pre compression and drug release properties increase with powder excipient ratios ( R ) from 5:1 to 50:1. A linear relationship exists between the liquid load factors $\mathrm{L}_{\mathrm{f}}$ and the reciprocal powder excipient ratios $(1 / \mathrm{R})$ required to produce acceptable flowing and readily compressible liquid / powder admixtures. The linear relationship between $\mathrm{L}_{\mathrm{f}}$ and the $1 / \mathrm{R}$ plot of liquisolid systems possesses $\mathrm{Y}$ intercept and slope equal to the $\Phi$ values of the cellulose carrier powder and silica coating material (Javadzadeh et al., 2007)

Liquisolid system dissolution rate profiles are affected by powder excipients ratio $\mathrm{R}$ in which results exhibited within the 5 minutes of the dissolution process against the $r$ values 5 to 20 range $R$ values. The dissolution rates increased almost proportionally to $\mathrm{R}$ until reaching an apparent maximum plateau at powder excipient ratios greater than 20 .

Lower $\mathrm{R}$ values of liquisolid compacts contain relatively smaller amounts of carrier powder (cellulose), a large amount of fine coating particles (silica), and the ratios of their liquid medication per powder substrate are relatively higher. From the low liquid / powder ratios, a high presence of cellulose and low presence of silica may be directly associated with enhanced wicking, disintegration, and degradation properties. Low $\mathrm{R}$ values should justifiably display relatively poor dissolution profiles. After disintegration, low $\mathrm{R}$ values of liquisolid tablets are overloaded with liquid medication producing the primary particles.

On other hand, in some cases, the drug diffusion through the primary particles may be rapid and might lead to overwhelming (solubility- wise) of the stagnant dissolution layers with drug. After maximum levels of dissolution are reached at 35 to $45 \mathrm{R}$ values, a slight gradual decrease of dissolution rate occurs with increasing powder excipient ratios. For $\mathrm{R}$ values higher than 50 , they may be attributed to the slower diffusion of the liquid medication through the numerous porous carrier powder particles into which the drug solution has been embedded during the formulation process. To determine the effect of different type of car- riers such as Avicel, lactose, starch or sorbitol, dissolve in solution containing $10 \% \mathrm{w} / \mathrm{w}$ of drug in liquid medication. Carriers show the potential to absorb the liquid medication. Large amounts of these carriers are necessary for regenerating liquid medication to dry looking and non adherent powder. Avicel ${ }^{\circledR} \mathrm{PH} 102$ showed better results, due to its large specific area in comparison with other carriers such as lactose and starch (Javadzadeh et al., 2007).

Type of carrier might affect the unit size of liquisolid tablets. Higher Avicel ${ }^{\circledR}$ PH 102 concentrations show uniform distribution of the drug by either adsorption onto or absorption into the carrier. Between the hydrogen groups, hydrogen bonds on adjacent cellulose molecules in Avicel ${ }^{\circledR}$ PH 102 may account exclusively for the strength and cohesiveness of compacts. Avice ${ }^{\circledR} \mathrm{PH} 102$ compressibility and compactness characteristics can be explained by the nature if crystalline cellulosic particles themselves which are held together by hydrogen bonds which when compressed, are deformed plastically and a strong compact is formed due to the extremely excessive number of surfaces brought into contact during the plastic deformation, and the strength of the hydrogen bonds are formed (Fahmy et al.,2008).

Surface active agent Polysorbate 80 was shown to facilitate wetting of drug particles by decreasing interfacial tension between dissolution medium and the tablet surface. Increase in the wetting properties of liquisolid tablets by the dissolution media is one of the main reasons for the dissolution rate enhancement. High $\mathrm{R}$ values 30 to 60 evidence better uniform distribution of the drug in the carrier material (Tayel et al., 2007).

\section{PRE COMPRESSION STUDIES}

Polymorphic changes of the drug are important factors that may affect the dissolution rate and bio availability. Prior to the compression of the formulation into tablets, and in order to ensure the suitability and selection of excipients, various studies were performed including Differential Scanning Calorimetry (DSC), X-Ray Powdered Diffraction (XRPD), and Scanning Electron Microscopic studies. In order to assess the thermo tropic properties and the thermal behaviors of the drug, carriers such as Avicel ${ }^{\circledR}$ PH 102, coating material such as Aerosil ${ }^{\circledR}$ PH 200 as well as liquisolid tablets were used.

Yousef Javadzadeh et al. (2008) suggested that the liquisolid technique has the potential to be optimized for the reduction of drug dissolution rate and thereby production of sustained release systems. The results also showed that wet granulation had a marked impact on reducing dissolution release rate of propranolol $\mathrm{HCl}$ from liquisolid formulations (Javadzadeh et al., 2008). 
Rania et al. (2008) reported that famotidine liquisolid tablets reached a maximum result within 30 minutes and compared to conventional tablets showed higher a dissolution pattern in both rate and extent of drug. Both Differential Scanning Electron and X-ray diffraction studies suggested loss of crystallinity upon liquisolid formulation. This was further confirmed by Scanning Electron Microscopic studies indicating that even though the drug existed in a solid dosage form, it is held within the powder substrate in a solubilized, almost molecularly dispersed state, which contributed to the enhanced drug dissolution properties. In addition, the selected optimal formula released $78.36 \%$ of its content during the first $10 \mathrm{~min}$ which is $39 \%$ higher than that of the directly compressed tablets. Further, the bioavailability study indicated that the prepared optimal liquisolid formula did not differ significantly from the marketed famotidine tablets concerning $\mathrm{C}_{\text {max }}, \mathrm{t}_{\max }$, and $\mathrm{AUC}(0-8)$ at $\mathrm{P}<0.05$.

Tayel et al. (2008) reported that Carbamazepine liquisolid tablets showed better dissolution rates, rapid disintegration and good wettability comparable to the generic product by using Avicel ${ }^{\circledR}$ PH 200 at a 20:1 carrier and coating material ratio.

In a study by Yousef Javadzadeh et al. (2007), in order to reduce the amounts of carrier and Aerosil PH 200 in liquisolid formulations, some additives namely, Poly Vinyl Pyrrolidone (PVP), Hydroxy Propyl Methylcellulose (HPMC) and Poly Ethylene glycol (PEG 35000) were added to liquid medication to increase loading factor. The results showed that the drug loading factor was increased significantly in the presence of additives. It was shown that microcrystalline cellulose had more liquid retention potential in comparison with lactose, and the formulations containing microcrystalline cellulose as a carrier showed a higher dissolution rate. By decreasing the ratio of microcrystalline cellulose to silica from 20 to 10 , an improvement in dissolution rate was observed. Further decrease in the ratio of microcrystalline cellulose: silica from 10 to 5 resulted in a significant reduction in dissolution rate. Increasing PVP concentration in liquid medication caused a dramatic increase in dissolution rate during the first $30 \mathrm{~min}$. The results showed that the dissolution rate of liquisolid tablets was not significantly affected by storing the tablets at $25^{\circ} \mathrm{C} / 75 \%$ relative humidity for a period of 6 months.

Yousef Javadzadeh et al. (2007) investigated the effect of type of carrier on dissolution rate of piroxicam from liquisolid tablets involving lactose, starch, sorbitol, Avicel. This study suggested that Avicel had more liquid retention potential due to the high specification area of Avicel $\left(1.18 \mathrm{~m}^{2} / \mathrm{g}\right)$ in comparison with other carriers lactose $\left(0.35 \mathrm{~m}^{2} / \mathrm{g}\right)$, starch $\left(0.6 \mathrm{~m}^{2} / \mathrm{g}\right)$ and sorbitol $\left(0.37 \mathrm{~m}^{2} / \mathrm{g}\right)$, but a larger amount of these carriers is necessary for converting liquid medication to dry-looking, non-adherent powder.

Javadzadeh et al. (2005) reported that surface active agent polysorbate 80 facilitated wetting of drug particles by decreasing interfacial tension between dissolution medium and tablet surface. Increase in the wetting properties of liquisolid tablets by the dissolution media is one of the main reasons for the piroxicam dissolution rate enhancement.

Ali et al. (2005) found that, from the indomethacin liquisolid tablets, the amount of drug release at $\mathrm{pH} 7.2$ is greater than that released at $\mathrm{pH} 1.2$ due to the higher solubility of indomethacin at higher $\mathrm{pH}$ values.

Khaled et al. (2001) reported that hydrochlorothiazide liquisolid tablets showed significantly greater extent of absorption than commercial tablets. Therefore, this formulation of the drug has the potential to be considered for human study in order to be manufactured on a large scale.

Studies by Spireas et al.(1999) reported it is quite possible that liquisolid compacts might exhibit more consistent and enhanced absorption characteristics compared to commercial methyclothiazide tablets .

Spireas et al. (1998) concluded that the dissolution rate of prednisolone from the liquisolid compacts is independent of the dissolution volume, whereas direct compressed tablets of the micronized drug display declining dissolution rates with decreasing volumes of the dissolving medium.

In a study on hydrocortisone liquisolid tablets, Spireas et al. (1998) reported that, due to their increased wetting properties and surface of drug available for dissolution, these tablets demonstrated significantly higher drug release rates than those conventionally made, direct compressed marketed products. Also, these authors observed that the drug release superiority of liquisolid tablets is inversely proportional to the aqueous solubility of the contained drug. Liquisolid tablets demonstrated significantly faster drug dissolution patterns in small volumes of dissolution media such as $450 \mathrm{~mL}$ and $300 \mathrm{~mL}$

Studies by Javadzadeh Y., et al. (2009) provide evidence that among the different grades of MCC evaluated, compacts containing MCC ${ }^{\circledR} \mathrm{PH} 101$ and 102 showed better hardness, friability and dissolution profile. Aging had no effect on hardness or dissolution profile of liquisolid tablets prepared from MCC PH 101. Finally, this study reported that MCC PH 101 is a suitable carrier for preparing liquisolid systems in terms of having acceptable flowability, friability, hardness, and dissolution profile.

Tiong N et al. (2009) reported that naproxen properties were changed by adding non-volatile hydrophilic 
solvent, leading to an increase in dissolution properties and possibly oral bio-availability.

\section{CONCLUSION}

In conclusion, Liquisolid technology was effective for improving dissolution rate as well as bio-availability in practically water insoluble drugs with non-volatile solvents. The technique also sustained the drug release properties of the water soluble drugs by using suitable biodegradable polymers with appropriate excipient ratios.

\section{REFERENCES}

AGUIAR,A. J.; ZELMER, A.J.; KINKEL,A.W. Deagglomeration behavior of relatively insoluble benzoic acid and its sodium salt. J. Pharm. Sci., v.56, p.1243-1252. 1979.

AMRIT KARMARKAR, B.; INDRAJEET GONJARI, D.; AVINASH HOSMANI, H.; PANDURANG DHABALE, N.; SATISH BHISE, B. Dissolution rate enhancement of fenofibrate using liquisolid tablet technique. Lat. Am. J. Pharm. v.28, p.219-225, 2009.

AMRIT KARMARKAR, B.; INDRAJEET GONJARI, D.; AVINASH HOSMANI, H.; PANDURANG DHABALE, N.; SATISH BHISE, B. Liquisolid tablets: a novel approach for drug delivery. Int. J. Health Res., v.2, p.45-50, 2009.

EBERT, W. R. Soft gelatin capsules: unique dosage form. Pharm. Tech., v.1, p.44-50, 1977.

FINHOLT, P.; SOLVANG, S. Dissolution kinetics of drugs in human gastric juice the role of surface tension. J. Pharm. Sci., v.57, p.1322-1326, 1968.

HITENDRA MAHAJAN, S.; MANOJ DHAMNE, R.; SURENDRA GATTANI, G.; ASHWINI. RASAL, D.; HANNAN SHAIKH, T. Enhanced dissolution rate of glipizide by a liquisolid technique. Int. J. Pharm. Sci. Nanotech., v.3, p.4-8, 2011.

JAVADZADEH, Y.; JAFARI-NAVIPOUR, B.; NOKHODCHI, A. Liquisolid technique for dissolution rate enhancement of a high dose water - insoluble drug (carbamazepine). Int. J. Pharm., v.341, p.26-34. 2007.

JAVADZADEH, Y.; MUSSALREZAEI, L.; NOKHODCHI, A. Liquisolid technique as a new approach to sustain propranolol hydrochloride release from tablet matrices. Int. J. Pharm., v.362, p.102-108, 2008.
JAVADZADEH, Y.; SIAHI, M. R.; ASNASHARI, S.; NOKHODCHI, A. An investigation of physicochemical properties of piroxicam liquisolid compacts. Pharm. Dev. Tech., v.12, p.337-343. 2007.

JAVADZADEH, Y.; SIAHI, M. R.; BARZEGAR JALALI, M.; NOKHODCHI, A. Enhancement of dissolution rate of piroxicam using liquisolid compacts. Farmaco, v.60, p.361-365, 2005.

JAVADZADEH, Y.; SHARIATI, H.; MOVAHHED-DANESH,E .; NOKHODCHI , A. Effect of some commercial grades of microcrystalline cellulose on flowability, compressibility and dissolution profile of piroxicam liquisolid compacts. Drug Dev. Ind. Pharm., v.35, p.243-251, 2009.

KAPSI, S.G; AYREYS, J.W. Processing factors in development of solid solution formulation of itraconazole for enhancement of drug dissolution behavior of water insoluble drugs. $J$. Pharm. Sci., v.76, p.744-752. 2001.

KARMARKAR,A.B. Effect of Ceolus KG-802 on the dissolution rate of fenofibrate liquisolid tablets: Preformulation and formulation development studies. Drug Discov. Ther., v.4, p.493-498,2010.

KHALED, A.; ASIRI, Y. A.; EL-SAYED,Y. M. In vivo evaluation of hydrochlorothiazide liquisolid tablets in beagle dogs. Int. J. Pharm., v.222, p.1-6, 2001.

KHALID EL-SAY, M.; AHMED SAMY, M.; MOHAMEDFETOUH, I. Formulation and evaluation of rofecoxib liquisolid tablets. Int. J. Pharm. Sci. Rev. Res., v.3, p.135-142, 2010.

KHALID EL-SAY, M.; AHMED SAMY, M.; MOHAMED FETOUH, I. Optimization of rofecoxib liquisolid tablets usingbox-behnken design and desirability function. $J$. Pharm. Res., v.3, p.2388-2392,2010.

LIAO, C. C. Physicochemical properties of selected powdered drug solutions. New York, 1983. p.95-176. [Ph.D. thesis. St.John's University].

LIN, S. L.; MENIG, J.; LACHMAN, L. Interdependence of physiological surfactant and drug particle size on the dissolution behavior of water insoluble drugs. J.Pharm. Sci., v.57, p.2143-2146, 1968. 
MARTIN, A. N.; SWARBBRICK, J; CAMMARATA, A. Physical pharmacy. Philadelphia: Lea \& Febiger, 1983. p.445-468.

NOKHODCHI, A.; JAVADZADEH, Y.; SIAHI, M.R.; BARZEGAR-JALALI, M. The effect of type and concentration of vehicles on the dissolution rate of a poorly soluble drug (indomethacin) from liquisolid compacts. $J$. Pharm. Sci., v.8, p.18-25. 2005.

RANIA, H. F.; KASSEM, M.A. Enhancement of famotidine dissolution rate through liquisolid tablets. Formulation. In vitro and in vivo evaluation. Eur. J. Pharm. Biopham., v.69, p.993-1003, 2008.

SHASHIDHER, B.; SANDEEP KUMAR, G. Enhancement of solubility and dissolution rate of frusemide through liquisolid technique. Pharm. Lett., v.2, p.321-328, 2010.

SPIREAS, S. Liquisolid systems and methods of preparing same. United State Patent 6423,339 B1, 2002.

SPIREAS, S. S.; JAROWSKI, C.I.; ROHERA, B.D. Powder solution technology: principles and mechanism. Pharm. Res., v.9, p.1351-1358, 1992.
SPIREAS, S.; WANG, T.; RAKESH,G. Effect of powder substrate on the dissolution properties of methyclothiazide liquisolid compacts. Drug Dev. Ind. Pharm., v.25, p.163$168,1999$.

SPIREAS, S.; SADU, S. Enhancement of prednisolone dissolution properties using liquisolid compacts. Int. J. Pharma., v.166, p.177-188, 1998.

SPIREAS, S.; SADU, S.; GROVER, R. In vitro release evaluation of hydrocortisone liquisolid tablets. J. Pharm. Sci, v.87, p.867-872, 1998.

TAYEL, S.A.; SOLIMAN, I. I.; LOUIS, D. Improvement of dissolution properties of carbamazepine through application of the liquisolid tablet technique. Eur. J. Pharm. Biopharm., v.69, p.342-347,2007.

TIONG, N.; ELKORDY, A. A. Effects of liquisolid formulations on dissolution of naproxen. Eur. J. Pharm. Biopharm., v.73, p.373-384, 2009.

Received for publication on $26^{\text {th }}$ November 2010 Accepted for publication on $18^{\text {th }}$ April 2011 